\title{
Weibull Analysis for Constant and Variant Stress Behavior Using the ALT Method for Single Stress and the Taguchi Method for Several Stress Variables
}

\author{
Manuel R Piña Monarrez* \\ Department of Industrial and Manufacturing, Universidad Autónoma de Ciudad Juárez, México
}

Submission: January 22, 2018; Published: April 18, 2018

*Corresponding author: Manuel R Piña Monarrez, Department of Industrial and Manufacturing, Engineering and Technological Institute, Universidad Autónoma de Ciudad Juárez, México; Email: manuel.pina@uacj.mx

Abstract

The article presents the advances on Weibull analysis which let practitioners to plan the data collection test, to fit the Weibull parameters directly from the applied stress values, to determine the capability indices cp and cpk, and to formulate the control charts to monitor the Weibull parameters. Thus, the theoretical background and formulation as well as numerical examples for constant and variant stress behavior are given. Also the references where the discussed methods were mathematically formulated and numerically applied are provided.

Keywords : Weibull demonstration test plan; Accelerated life time analysis; Taguchi method; Sample size; Capability indices

Abbreviations : NHPP: Non-Homogenous Poisson Process; T: Temperature; ALT: Accelerated Life Time Data; Mn: Manganese; Mg: Magnesium; HMPC: Hot Mill Pass Counts; CMR: Cold Mill Reduction Rate

\section{Introduction}

Since the Weibull distribution is the best probability density function (pdf) used to model the behavior of a quadratic form $[1,2]$, as they are any response surface model [3], the stress and strain matrix used in mechanical and structural analysis [4], the covariance matrix used in principal component analysis [5], and the branching process behavior [1]. Then an understanding of the Weibull distribution features is needed. Based on the above, this paper presents the advances on Weibull analysis to perform it from the planning data collection phase to the monitoring process phase. Therefore, the papers' structure is as follows. In section 2 , the general new background of the Weibull distribution and the formulation to determine the sample size to perform a zero failure Weibull demonstration test plan are presented. In section 3 , the formulation to determine the capability $c p$ and cpk indices and the formulation of the control charts which let us to monitor the estimated Weibull parameters are given. In section 4 , the formulation to fit the Weibull shape $\beta$ and scale $\eta$ parameters directly from the applied stress values is presented. In section 5 a numerical example for constant stress is given. In section 6 a numerical example for variant stress is given. Finally, in section 7 the conclusions are presented. The analysis is as follows:

\section{Weibull distribution background and sample size}

The two parameter Weibull distribution [6] is given by

$$
f(t)=\frac{\beta}{\eta}\left(\frac{t}{\eta}\right)^{\beta-1} \exp \left\{-\left(\frac{t}{\eta}\right)^{\beta}\right\}
$$

From Eq. (1), the Weibull reliability function is given by

$$
R(t)=\exp \left\{-\left(\frac{t}{\eta}\right)^{\beta}\right\}
$$

And since the Weibull distribution is generated by a nonhomogenous Poisson process (NHPP) [7] sec 4.3, then the Weibull risk function depends on the time also. Thus, the mean power function of the related NHPP in Weibull analysis is used as the cumulative hazard risk function $H(t)$. From Eq. (2), $H(t)$. is given by

$$
H(t)=\left(\frac{t}{\eta}\right)^{\beta}=-\ln (R(t))
$$


In Weibull analysis Eq. (3) is of special interest because for the desired time $t$ and known $\beta$ value the effect that the stress variable $s$ has on the estimated $R(t)$ index is given by the linear relationship between $\eta$ and $\mathrm{s}$ as

$$
\eta=\exp \left\{\alpha^{t} Z\right\}
$$

In Eq. (4), $\alpha$ is a regression coefficient vector $\alpha=\left\{b_{0}, \beta_{1}, \ldots, \beta_{p}\right\}$ and $Z$ is a nxp matrix which contains the $k$ stress variables $\left(s_{1}, s_{2}, \ldots, s_{k}\right)$ and $p$ represents the $\mathrm{k}$ variables effects plus the possible effects generated among the $k$ variables (e.g. interaction and quadratic effects). Also note from Eq. (2), that for known $t$ and $\beta$ values, the $\eta$ value which corresponds to a desired $R(t)$ index is completely addressed and it is given as

$$
\eta=\frac{t}{[-\ln (R(t))]^{1 / \beta}}
$$

On the other hand, because by setting $H(t)=1$ in Eq. (3) the sample size $n$ which have to be tested to accurately estimate $\eta$, was derived in [8]. as

$$
n=\frac{-1}{\ln (R(t))}
$$

Then, from Eq. (5 and 6) $\eta$ in terms of $n$ is given by

$$
\eta=n^{1 / \beta} t
$$

Eq. (6) is too important in Weibull analysis because by using it in Eq. (7), the $\eta$ value which corresponds to any feasible or desired $t(t>0), \quad \beta(\beta>0)$ and $R(t)$ values is completely determined without any experimentation or observed lifetime data. As an example suppose we desire to determine the $\eta$ value which corresponds to $R(t)=0.9535$ for $t=1500 \mathrm{hrs}$ and $\beta=3$. Thus from Eq. (6) $n=21$ and by using it with $\beta=3$. and $t=1500 \mathrm{hrs}$ in Eq. (7), $\eta=21^{1 / 3} 1500=4138.38 \mathrm{hrs}$. Hence, because the value estimated in Eq. (6) completely determine $\eta$, then this $n$ value also represents the sample size which has to be tested without failures in order to accurately estimate the minimum $\eta$ value for which the tested element will present at least the desired reliability index. As a simple example suppose we have to demonstrate a product fulfills with $R(t)=0.95$ for $t=1500$ hrs Thus, because from Eq. (6) $n=19.4957$, then 19 parts has to be tested by $1500 \mathrm{hrs}$ each and one part has to be tested by $0.4957(1500)=743.595 \mathrm{hrs}$. It is to say, $n$ in Eq. (6) is not a discrete value, instead it is continuous and it represents the times the desired time $t$ has to be tested in order to demonstrate the tested product fulfills at least with the desired $R(t)$ index. Now we know $n$ in Eq. (6) represents the right sample size to design a zero failure Weibull demonstration test plan, let present the capability indices and the corresponding control charts.

\section{Weibull capability indices and control charts}

When the Weibull analysis is being performed for example in the quality field or in an improvement process, the related capability index $c p$ and ability index $c p k$ are of interest. Fortunately by using the log-mean $\left(\mu_{x}\right)$ and the log-standard $\left(\sigma_{x}\right)$ deviation parameters of the observed (or expected) failure times, as was demonstrated in [9] and [10], the Weibull $c p$ and $c p k$ indices can both be estimated. In particular, they are formulated based on the direct relationships between the Weibull $\beta \eta$ parameters with the $\log \mu_{x}$ and parameters. These relationships are

$$
\begin{gathered}
\beta=\frac{R \sigma_{y}}{\sigma_{x}} \\
\eta=\exp \left\{\mu_{x}-\frac{\mu_{y}}{\beta}\right\}
\end{gathered}
$$

In Eq. (8), $R$ is the multiple correlation coefficient between the responses $Y_{i}$ values and the logarithm of the failure times $\left(X_{i}=\ln \left(t_{i}\right)\right.$ ) (here after, by supposing data is Weibull, $R=1$ ). In Eq. (9) $\mu_{y}$ and in Eq. (8) $\sigma_{y}$ are the mean and the standard deviation of the response vector given by

$$
Y_{i}=\ln \left(-\ln \left(1-F\left(t_{i}\right)\right)\right)=b o+B X_{i} \text { (10) }
$$

In Eq. (10), $b_{0}$ and $B$ are parameters to be estimated and the cumulated failure probability $\left(F\left(t_{i}\right)\right)$ is given by the median rank approach here approximated by the well-known Bennard formula as

$$
F(t)=\frac{i-0.3}{n+0.4}
$$

Therefore, based on the $\sigma_{y}$ parameters given in Eqs.(8 and 9), the corresponding $c p$ and $c p k$ indices are

$$
\begin{aligned}
& C_{p W}=\frac{(U S L-L S L)}{6 \sigma_{x}} \\
& C_{p k W}=\min \left(C_{p u}, C_{p l}\right)=\min \left(\frac{U S L-\mu_{x}}{3 \sigma_{x}}, \frac{\mu_{x}-L S L}{3 \sigma_{x}}\right)(13)
\end{aligned}
$$

In Eq. (10 and 11), USL and LSL are the upper and lower product's specifications limits measured in time units. And if they are unknown, then the minimum and maximum expected lifetimes of the Weibull analysis can be used to estimate them. These maximum and minimum lifetimes are estimated from Eq. (10) by using the $Y_{n}$ maximum element to estimate the maximum lifetime and by using the $Y_{i}$ minimum element to estimate the minimum lifetime as

$$
t_{i}=\exp \left\{\frac{Y_{i}+b_{0}}{\beta}\right\}=\exp \left\{\frac{Y_{i}+\beta \ln (\eta)}{\beta}\right\}
$$

On the other hand, based on the facts that

1) In Weibull analysis the $R(t)$ index is completely defined by the $\beta$ and $\eta$ parameters, and 
2) The $\beta$ and $\eta$ are determined by the $\left(\sigma_{x}\right)$ [Eqs. 8 and 9].

Then in [12], the Weibull control charts to monitor $\beta$ and $\eta$ were formulated by setting $\left(\sigma_{x}\right)$ as the upper control limit to monitoring. And by setting $\left(\mu_{x}\right)$ as the lower control limit to monitoring $\eta$. Here it is too important to highlight because represent the central parameters in logarithm scale, then in the case where we are analyzing several variables can be estimated by using the Taguchi method as it is made in [11]. And of course, once they had been already estimated, they can be used in Eqs. ( 8 and 9) to estimate the corresponding $\beta$ and $\eta$ parameters, as well as in Eqs. (12 and 13) to determine the corresponding capability indices; for details see [12]. Now let present how to estimate $\beta$ and $\eta$ directly from the applied stresses values.

\section{Estimation of $\beta$ and $\eta$ directly from the stresses values}

Since in Weibull analysis $\beta$ and $\eta$ completely determine the estimated $R(t)$ index, and because $\beta$ represents the aging of the analyzed system, then their accurate estimation is needed. Fortunately, as demonstrated in [4] both $\beta$ and $\eta$ can be directly estimated from the maximum and minimum applied stresses values. The estimation is made by using the eigen values of the analyzed quadratic form as the maximum and minimum applied stress values. As examples of quadratic forms suppose we are analyzing a biological phenomenon by using a response surface model given by

$$
Y=b_{0}+\beta_{1} X_{1}+\beta_{2} X_{2}+\beta_{11} X_{1}^{2}+\beta_{22} X_{2}^{2}+\beta_{12} X_{1} X_{2}
$$

Thus, its quadratic form is $Q=\left[\begin{array}{cc}\beta_{11} & \beta_{12} / 2 \\ \beta_{21} / 2 & \beta_{22}\end{array}\right]$. Similarly suppose from the stress analysis we know the normal stresses and the shear stress $\tau_{x y}$ values. Hence, the stress

quadratic form is $Q=\left[\begin{array}{cc}\sigma_{x} & \tau_{x y} \\ \tau_{y x} & \sigma_{y}\end{array}\right]$. As a third example of quadratic form suppose in a principal component (pc) analysis we know the variance and the covariance among the variables. Thus, the pc quadratic form is $Q=\left[\begin{array}{cc}\sigma_{11} & \sigma_{1} \sigma_{2} \\ \sigma_{2} \sigma_{1} & \sigma_{22}\end{array}\right]$ Therefore based on [4], by using the eigen values $\lambda_{1}$ and $\lambda_{2}$ of the quadratic form $Q$, the $\beta$ value is given as

$$
\beta=\frac{-4 \mu_{y}}{\ln \left(\lambda_{1} / \lambda_{2}\right)}
$$

In Eq. (16) $\mu_{y}$ is the mean of the response vector defined in Eq. (10). And because the log-mean is directly given as the square root of the determinant of the $Q$ matrix (for details see sec. 2.2 in [4]), then by using $\mu_{y}$ and the estimated $\beta$ values in Eq.(9), $\eta$ is directly estimated. It is to say from Eq. (16) $\beta$ is estimated and from Eq. (9) the corresponding $\eta$ value is estimated. Now let present the numerical examples.

\section{Numerical example for constant stress behavior}

In this section two numerical example with constant stress behavior are presented. In the case of single stress variable, the temperature (T) is used as the stress variable. And for several stress variables the Taguchi method is used.

\section{Analysis with a single stress variable}

Table 1: Data for single constant stress analysis.

\begin{tabular}{|c|c|c|c|}
\hline Stress & $\mathbf{3 9 3 K}$ & $\mathbf{4 0 8 K}$ & $\mathbf{4 2 3 K}$ \\
\hline \multirow{4}{*}{ Time } & 3850 & 3300 & 2750 \\
\cline { 2 - 4 } & 4340 & 3720 & 3100 \\
\cline { 2 - 4 } & 4760 & 4080 & 3400 \\
\cline { 2 - 4 } & 5320 & 4560 & 3800 \\
\cline { 2 - 4 } & 5740 & 4920 & 4100 \\
\cline { 2 - 4 } & 6160 & 5280 & 4400 \\
\cline { 2 - 4 } & 6580 & 5640 & 4700 \\
\cline { 2 - 4 } & 7140 & 6120 & 5100 \\
\cline { 2 - 4 } & 7980 & 6840 & 5700 \\
\cline { 2 - 4 } & 8960 & 7680 & 6400 \\
\hline
\end{tabular}

In this section let used data published in [13]. The $n=10$ collected lifetimes are given in Table 1. Data corresponds to an accelerated life time data (ALT) subjected to a single stress variable (temperature in Kelvin degrees). The normal level is $N=323 K$ and the accelerated levels are lower $L=393 K$, middle $M=408 K$ and higher $H=423 K$. Thus, because data of Table 1 is an ALT data, then first the $\eta$ parameter of the normal level $T=323 \mathrm{~K}$ has to be determined. And because the stress variable is a fixed temperature value, then the life/stress Arrhenius models is used. (If the stress is a range of temperature, then the Coffin Mason model should be used). The Arrhenius model ([14] sec.5.5.1) is given as

$$
\eta_{i}=L\left(T_{i}\right)=C e^{\frac{B}{T_{i}}}
$$

In Eq. (17) $C$ and $B$ are parameters to be estimated. Thus, by using Eq. (1) and Eq. (17) in the ALTA software, the estimated Arrhenius parameters are $\beta=4.2916, C=58.9848$ and $B=1861.6187$. As a consequence, by using the estimated $C$ and $B$ values with $\mathrm{T}=323$ in Eq. (17), the normal scale parameter is $\eta=18784.83$ hrs. Therefore the Weibull distribution of the normal setting is $W(4.2916,18784.83)$. On the other hand, the values which corresponds to $R(t)=0.9535$ are estimated by using $\mu_{y}=-0.545624$ and $\sigma_{y}=1.1751169$. were estimated by using $R(t)=1-F(t)=0.9535$ in Eq.(10)

Therefore, from Eq. (9), $\mu_{x}=9.7136675$ and from Eq. (8) $\sigma_{x}=0.2738179$. And because $n=-1 / \ln (0.9535)=21$, then $Y_{n}=\ln (-\ln (1-((21-0.3) / 21.4)))=1.22966, \quad Y_{1}=-3.403483$. Thus, from Eq. (14) $\mathrm{UCL}=10.12733$ and $\mathrm{LCL}=9.04774$. And as a consequence from Eq.(12), cpw $=0.657118$. And from Eq. (13) $\operatorname{cpkw}=\min (0.5035,0.8106)=0.5035 .$. Finally, $\sigma_{x}=0.2738179$ has to be set as the maximum allowed value to monitor $\beta$. Similarly $\mu_{x}=9.7136675$ has to be set as the lower allowed value to monitor $\eta$. Now let present the multivariate analysis. 


\section{Analysis with several stress variables}

In this section, the analysis is based on [11]. The used data given in Table 2 was first published in [15]. The analyzed factors were the aluminum-alloy content in manganese ( $\mathrm{Mn})$ and magnesium (Mg) as well as hot mill pass counts (HMPC) and cold mill reduction rate (CMR). $\mathrm{Mn}, \mathrm{Mg}$, and CMR were measured in percentage units until dome yielded $1 \mathrm{~mm}$ on a constantly applied external pressure of 500psi. From the Taguchi analysis ([11] sec.3.3.2) the robust level is $\mathrm{Mn}=1.6, \mathrm{Mg}=1.8, \mathrm{HMPC}=35$, and $\mathrm{CMR}=30$. The found robust's log-parameters are $\mu_{x}=3.5442$ and $\mu_{x}=0.024141$. Thus, from Eq.(8) $\beta=40.82715$ and from Eq.(9) $\eta=35.0307$.

Table 2: Taguchi data for constant stress analysis.

\begin{tabular}{|c|c|c|c|c|c|c|c|c|}
\hline \multicolumn{4}{|c|}{ Control Factors } & \multicolumn{5}{|c|}{ Replicates (Life Data) } \\
\hline $\mathrm{Mn}$ & $\mathrm{Mg}$ & HMP C & CMR & R 1 & $\mathrm{R} 1$ & R3 & R4 & Rs \\
\hline 0.4 & 0.6 & 25 & 30 & 17 & 21 & 23 & 18 & 16 \\
\hline 0.4 & 1.2 & 30 & 45 & 23 & 24 & 21 & 20 & 18 \\
\hline 0.4 & 1.8 & 35 & 60 & 23 & 26 & 27 & 25 & 22 \\
\hline 1 & 0.6 & 30 & 60 & 15 & 13 & 17 & 17 & 14 \\
\hline 1 & 1.2 & 35 & 30 & 20 & 21 & 22 & 22 & 20 \\
\hline 1 & 1.8 & 25 & 45 & 25 & 27 & 26 & 21 & 24 \\
\hline 1.6 & 0.6 & 35 & 45 & 24 & 25 & 24 & 22 & 22 \\
\hline 1.6 & 1.2 & 25 & 60 & 28 & 26 & 28 & 25 & 23 \\
\hline 1.6 & 1.8 & 30 & 30 & 32 & 34 & 31 & 36 & 33 \\
\hline
\end{tabular}

On the other hand, because in [11] $R(t)=0.90$ was used, then in this case $\mu_{\mathrm{y}}=-0.52311$ and $\sigma_{x}=1.115 .34$. Hence, from Eq. (6) $n=-1 / \ln (0.90)=10$, and from Eq. (10) $Y_{n}=0.992689, \quad Y_{1}=-2.66384$. Similarly, from Eq. (14) UCL= 3.580539 and LCL=3.490978. And from Eq. (12) $C p_{W}=0.618321$. And from Eq. (13) $C p k_{W}=\min (0.501764,0.734878)=0.501764 \mathrm{~s} \quad$ Finally in a control chart, to monitoring $\beta \quad \sigma_{x}=0.024141$ represents the maximum allowed value. Similarly in a control chart to monitoring $\eta$ $\mu_{x}=3.5442$ represents the lower allowed value to be monitored. For details on how a Weibull analysis with several variables can be performed see [16]. Now let present the analysis for nonconstant stress variable.

\section{Numerical example for variable stress behavior}

In this section two numerical example with variable stress behavior are presented. In the first case data published in [4] is used to show how a product can be designed. In the second case data published in [17] is use to show how the ALT analysis can be used to analyze variable stress behavior. The analysis is as follows

\section{Variable stress analysis focused on product's design}

In this section the focus is on product's design. The analysis is performed following [4]. The objective consists on determining the $R(t)$ index of a mechanical or structural design subjected to the normal stresses $\sigma_{x}=100 \mathrm{mpa}, \sigma_{y}=70 \mathrm{mpa}$, and a shear stress $\tau_{x y}=40$ mpa. Thus, as mentioned in section 4, the stress matrix $Q$ which model the variant stress behavior is $Q=\left[\begin{array}{cc}100 & 40 \\ 40 & 70\end{array}\right]$.
From the $\mathrm{Q}$ matrix, the principal stresses or eigen values are $\sigma_{1}=127.72 \mathrm{mpa}$ and $\sigma_{2}=42.28 \mathrm{mpa}$. And as a consequence, the principal stress behavior is in the interval [42.28 - 127.72] mpa. And because in [4] $R(t)=0.9535$ was used, then from the $Y_{i}$ elements generated in Eq. (10), their mean is $\mu_{y}=-0.545624$ and their standard deviation is $\sigma_{y}=1.1751169$. Hence, by using the $\mu_{y}$ value and the $\sigma_{1}$ and $\sigma_{2}$ values in Eq. (16), the estimated Weibull shape parameter is $\beta=1.984693$. And by using the $\sigma_{y}$ and $\beta$ values in Eq. (8), $\sigma_{y}=0.592090$. Finally, because from the logarithm of the square root of the determinant of the stress matrix $Q, \mu_{x}=4.29707726$, then from Eq.(9), the Weibull scale parameter is $\eta=96.73676 \mathrm{mpa}$. Therefore the addressed Weibull family is $W(1.984693,96.7367)$. However, because in variable stress analysis, the stress variable instead of be a single value it is a range of stress values, then the addressed Weibull family only represents the stress distribution which models the stress range behavior as shown in Table 2 in [4]. As a consequence, in order to determine the $R(t)$ index of the analyzed product, the Weibull distribution which models the strength of the product to overcome the applied stress has to be determined. In [4] sec. 4.4 .2 , because the used material strength was $s y=400 \mathrm{mpa}$, then the addressed strength Weibull family is W(1.984693, 455.2318). Finally, by using both the stress and the strength distributions in the Weibull/Weibull stress/strength methodology ([18] chapter 4 to 6$)$, the stress/strength $R(t)$ index is given as

$$
R(t)=\frac{\eta_{S}^{\beta}}{\eta_{S}^{\beta}+\eta^{\beta}}
$$

In Eq. (18) $\beta=1.984693, \eta=96.73676$ and $\eta_{s}=455.2318$. As a consequence, the addressed stress/strength $R(t)$ index 
is $R(t)=95.58$. It is to say a product designed with $\mathrm{sy}=400 \mathrm{mpa}$ performing in a variant stress range of $[42.28,127.72] \mathrm{mpa}$ will present a $R(t)=95.58$. [4].

On the other hand, although in variable stress analysis the $c p$ and $c p k$ indices defined in Eqs. (12 and 13) are not defined for the Weibull/Weibull stress/strength function yet, the related control charts can be used to monitor the stress and the strength Weibull parameters. Thus, $\sigma_{x}=0.592090$ should be used as the maximum value to monitor the common $\beta$ value, and $\mu_{x}=4.29707726$ should be used as the maximum value to monitor the stress $\eta$ value. Also observe because here represents the stress distribution it was set as the maximum allowed value. On the other hand, when it represents the strength distribution, it should be set as the minimal allowed value. Finally, the value to monitor the strength $\eta_{S}$ value is determined by using $\mu_{y}=-0.545624, \beta=1.984693$ and $\eta_{S}=455.2318$ in Eq. (9). The estimated value to be monitored as the minimal allowed stress value is $\mu_{x}=5.845891$. Now let present the stress variable case using ALT data.

\section{Variable stress analysis by using ALT data}

In this section the focus is to show how the ALT analysis can be used to analyze variable stress behavior. The used data was published in [17]. Data represents a set of 65 ball bearings tested at loads of 3500, 3800 and 4500 pounds. (Observe because the stress variable behavior by itself represents the normal stress behavior, then in the ALT analysis no extrapolation is needed). In the analysis the lognormal distribution with log parameters $\mu_{x}=7.6$ and $\sigma_{x}=0.4$ were used to represent the stress variable behavior. And the Weibull distribution was used to represent the corresponding life times behavior of the ball bearings. From the collected 65 ALT data, the Weibull $\beta$ parameter and the $K$ and $n$ parameters of the used inverse power model (IP)were estimated. The IP model [14] is given by

$$
L(V)=\frac{1}{K V^{n}}
$$

In Eq. (19), V represents the stress variable (pounds in this case). From the ALT analysis in [17], $\beta=1.847127, \mathrm{n}=3.624360$, and $\mathrm{K}=7.166599 \mathrm{E}-18$. Then 21 stress values in the interval $[1000,4000]$ punds were selected, and by using them with the estimated IP parameters in Eq. (19) $21 \eta$ values were predicted. Then by using $\beta=1.847127$, and the 21 estimated $\eta$ values in Eq. (2), the corresponding $R(t)$ indices for $t=30000$ were estimated and used in Eq. (10) to determine the corresponding $21 Y_{i}$ elements. Finally by using the logarithm of the 21 stresses values and the estimated $21 Y_{i}$ elements, in a regression, the Weibull parameters of the strength distribution were estimated. The fitted Weibull parameters of the strength distribution are $\beta=6.694655$, and $\eta=3126.926895$. Therefore, the addressed Weibull strength distribution is $\mathrm{W}(6.694655,3126.926895)$. Here it is too important to observe the addressed Weibull family only represents the behavior for fixed $t=30000$, if other $t$ value is desired the above process has to be repeated [17]. Finally, by using the lognormal stress distribution and the Weibull strength distribution in the lognormal/Weibull stress/strength analysis [18], the corresponding $R(t)$ index was determined. The found $R(t)$ index is $R(t)=0.7954$; [17].

On the other hand, although the $c p$ and $c p k$ indices are not defined for the lognormal/Weibull stress/strength analysis yet, the monitoring process of the addressed stress and strength parameters can be performed. This can be done because both the lognormal and the Weibull distribution are based on the log parameters. Therefore the lognormal parameter $\mu_{x}=7.6$ can be monitored by set $\mu_{x}=7.6$ as the maximum allowed stress level in a control chart. Note it was set as the maximum because it represents the stress distribution. It is to say, because In the stress/strength analysis the higher the stress value the lower the $R(t)$ index then it has to be set as the maximum allowed value. Similarly, the log normal parameter can be monitored by using $\sigma_{x}=0.4$ as the maximum allowed value also [18].

On the other hand, because in the monitoring process the variables which determine the values are the process variables which have to be monitored. And because in order to correctly monitor the contribution that each variable has on the observe values have to be determined, then their contribution have to be determined also. Fortunately because $\mu_{\mathrm{x}}$ is directly given from the determinant of the $Q$ matrix as it is made in [4], then the decomposition method given in [19] can be used to determine the mentioned variable contributions. Also it is important to mention that the mathematical formulation to determine the value and the variables which determine its value, can be performed by partitioning the $Q$ matrix as it is made in [20]. Finally the value to monitor $\beta=1.847127$ is determined by using $\sigma_{y}=1.1751169$ in Eq. (8). The $\left(\sigma_{x}\right)$ value to be set in the corresponding chart as the maximum allowed value is $\sigma_{x}=0.636186$. And because $\eta=3126.926895$ and $\mu_{y}=-0.545624$, then from Eq. (9) the value to be set in the corresponding chart as the minimum allowed value to monitoring $\eta$ is $\mu_{x}=8.343197$.

Finally, it is important to note the estimated stress/strength $R(t)=0.7954$ index was not used to estimate $\mu_{y}=-0.545624$ and $\sigma_{y}=1.1751169$ instead $R(t)=0.9535$ was used. This is made in this way because in the stress/strength analysis, the stress distribution is independent of the strength distribution. Therefore, the generated stress/strength function only represents the effect that all possible stress values have over the all possible strength values [18].

\section{Conclusion}

In this paper the advances on the theoretical interpretation of the features of the Weibull distribution which let practitioners to perform an integral analysis from the test planning phase to the monitor phase are presented. This is made based on the fact the Weibull distribution efficiently model a quadratic form. Among the more important features given in this article are 
The Weibull parameters $\beta$ and $\eta$ are completely determined from the maximum and minimum eigen values of the analyzed quadratic form (see sec. 4).

Because the addressed $n$ value in Eq.(6) only depends on the desired $R(t)$ index, then the estimated $\mathrm{n}$ value is robust under any uncertainties of the used $t$ and $\beta$ values; and in particular observe from Eq. (61) in [4] that because this $n$ value also represent the base lifetime of any Weibull analysis, then Eq. (6) can be used to determine the expected lifetimes after any reliability improvement process.

The mean of the expected logarithm of the lifetimes is completely determined by the logarithm of the square root of the determinant of the analyzed quadratic form $Q$.

Although the Weibull $c p$ and $c p k$ indices are estimated by using the logarithm parameters because they are dimensionless they are efficient.

By monitoring the log-parameters the $\beta$ and $\eta$ parameters are completely controlled.

\section{References}

1. Segun Goh, Kwon HW, Choi MY (2014) Discriminaring between Weibull distributions and log-normal distributions emerging in branching processes. J Phys A Math Theor 47: 22.

2. Todinov MT (2009) Is Weibull the correct model for predicting probability of failure initiated by non-interacting flaws?. International Journal of solids and structures. 46(3): 87-901.

3. George E P, Box, Draper (1987) Empirical Model Building and Response Surfaces. Wiley Series in Probability and Mathematical Statistics 13: 978-0471810339.

4. Piña-Monarrez MR (2017) Weibull stress distribution for static mechanical stress and its stress/strength analysis. Qual Reliab Engng Int p. 1-16.

5. Peña D (2002) Análisis de Datos Multivariantes. McGraw Hill/ Interamericana de España.

6. Weibull W (1939) A statistical theory of the strength of materials. Proceedings, R Swedish Inst Eng Res 151: 45.
7. Rinne H (2009) The Weibull distribution a handbook. Chapman and Hall/CRC press, Boca Ratón, FL, USA.

8. Piña-Monarrez MR, Ramos LML, Alvarado IA, Molina ARD (2016) Robust sample size for weibull demonstration test plan. DYNA 2016 83: 52-57.

9. Piña-Monarrez MR, Ortiz YJF, Rodriguez BMI (2016) Non-normal capability indices for the Weibull and lognormal distributions. Qual Reliaby Engng Int 32:1321-1329.

10. Piña-Monarrez MR, Baro Tijerina M, Ortiz YJF (2017) Unbiased Weibull capability indices using multiple linear regression. Qual Reliaby Engng Int 33(8): 1915-1920.

11. Piña-Monarrez MR, Ortiz YJF (2015) Weibull and lognormal taguchi analysis using multiple linear regression. Reliability Engineering and System Safety 144: 244-53.

12. Piña-Monarrez MR (2016) Conditional Weibull control charts using multiple linear regression. Qual Reliab Eng Int 33(4): 785-791.

13. Vassiliou P, Metas A (2002) Understanding accelerated life-testing analysis, Reliability and Maintainability Symposium (RAMS), Tutorials $\mathrm{CD}$, Seattle, WA, USA.

14. Bagdonaviciius V, Nikulin M (2002) Accelerated life models, modeling and statistical analysis, Ed. Chapman and Hall/CRC, Boca Ratón, FL, USA.

15. Besseris GJ (2010) A methodology for product reliability enhancement via saturated-unreplicated fractional factorial designs. Reliab Eng Syst Saf 95:742-749.

16. Piña-Monarrez MR, Avila CC, Marquez LCD (2015) Weibull accelerated life testing analysis whit several variables using multiple linear regression, DYNA 82(191): 156-162.

17. http://www.weibull.com/hotwire/issue172/hottopics172.htm

18. Kececioglu D (2003) Robust Engineering Design-By-Reliability with Emphasis on Mechanical Components \& Structural Reliability. DEStech Publications 1: 722

19. Piña-Monarrez MR (2013) Practical Decomposition Method for T2 Hotelling Chart. International Journal of Industrial Engineering Theory Applications and Practice 20(5-6): 401-411.

20. Piña-Monarrez MR (2011) A New Theory in Multiple Linear Regression. International Journal of Industrial Engineering Theory Applications and Practice 18(6): 310-316.

\section{Your next submission with Juniper Publishers}

will reach you the below assets

- Quality Editorial service

- Swift Peer Review

- Reprints availability

- E-prints Service

- Manuscript Podcast for convenient understanding

- Global attainment for your research

- Manuscript accessibility in different formats

( Pdf, E-pub, Full Text, Audio)

- Unceasing customer service

Track the below URL for one-step submission

https://juniperpublishers.com/online-submission.php 